\title{
Metabolismo intermediario basal y postprandial en obesos de altura y de nivel del mar*
}

\author{
Basal and postprandial intermediary metabolism \\ in high altitude and sea level obese patients
}

\author{
Fausto Garmendia-Lorena ${ }^{1,3}$, Rosa Pando-Alvarez ${ }^{1}$, Yanet Mendoza-Muñoz ${ }^{2}$, William Torres -Damas ${ }^{l}$
}

\begin{abstract}
Resumen
Objetivos: Estudiar el metabolismo intermediario basal y postprandial en obesos de altura (OA), y compararlo con personas con peso normal de altura (NA) y con obesos de nivel del mar (ONM) Material y métodos: Se incluyó a 30 NA-, 39 obesos de altura OA (Cusco, 3,395 msnm.) y a 35 obesos de nivel del mar ONM (Lima, $150 \mathrm{msnm}$ ), de ambos géneros, de 40 a 70 años de edad. En ayunas se midió glucosa (G), triglicéridos (Tg), colesterol total (CT), HDL, insulina (I), ácidos grasos no esterificados (AGNE), el colesterol VLDL, LDL, NoHDL y coeficiente HOMA fueron calculados y luego durante 6 horas después de un desayuno mixto estándar se determinó G, I, Tg y AGNE. Resultados: En condiciones basales los OA presentaron HDL más bajo y Tg y AGNE más altos que los NA y AGNE más altos que los ONM. Durante el período postprandial, los OA tuvieron valores más altos de I, Tg y NEFA que los NA y concentraciones más altas de Tg y AGNE que los ONM con mínimas diferencias en la G e I. Conclusiones: Los obesos de altura presentan resistencia a la insulina en comparación a los normales de altura y valores más altos de triglicéridos y 'NEFA que los obesos de nivel del mar.
\end{abstract}

Palabras clave: Altura, obesidad, metabolismo intermediario.

Abstract

Objectives: To study the basal and postprandial intermediary metabolism of obese high altitude dwellers (OHAD), and to compare with the intermediary metabolism of normal high altitude dwellers (NHAD) and with obese of sea level dwellers (OSLD). Methods: A total of 30 NHAD, 39 OHAD (Cusco, Perú, at 3395 meters above the sea level) and 35 OSLD (Lima, Perú, at 150 meters above the sea level), of both genders, 40 to 70 years old were included. Glucose (G), total cholesterol (TC), HDL cholesterol (HDL), triglycerides (Tg), insulin (I) and non-esterified fatty acids were measured in blood at fasting, Cholesterol VLDL,LDL, NOHDL and HOMA coefficient were calculated; thereafter and during 6 hours after ingestion of a standardized mixed meal G, I, Tg and NEFA were determined in blood. Results: At fasting HAOD had lower HDL and higher Tg and NEFA concentrations than HAND and higher NEFA than OSLD. During the postprandial period HAOD presented higher values of I, Tg and NEFA than HAN and higher values of Tg and NEFA than SLO with minor differences in G and I. Conclusions: HAO subjects in comparison to HAN present resistance to insulin and in relation to SLO higher values of Tg and NEFA

Keywords: High altitude, obesity, intermediarymetabolism.

\section{Introducción}

En trabajos anteriores, se demostró que el metabolismo intermediario tanto de hombres como de mujeres de altura es diferente al de grupos similares de nivel del mar tanto en condiciones basales como postprandiales ${ }^{(1,2)}$, en los que destacan la menor glicemia y las concentraciones mayores de Tg y NEFA. Tomando en cuenta estos antecedentes, se consideró importante llevar a cabo esta investigación en pobladores obesos de ambos géneros que viven en la altura, desde que la obesidad es considerada como una enfermedad crónica no trasmisible que predispone a otras condiciones que incrementan la morbimortalidad por alteraciones cardiovasculares. Se ha establecido que el medio ambiente de altitud, que se caracteriza por menores presiones barométrica y parcial de $\mathrm{O}^{2}$, determina una situación de hipoxia que modifica las funciones respiratoria, cardiovascular, hemática y metabólica del organismo. Desde $1939^{(3)}$ se conoce que el poblador normal de altura (PNA) tiene una glicemia menor que el poblador de nivel del mar (PNM), fenómeno comprobado por muchos otros investigadores $^{(4-13)}$, que no es dependiente del mayor hematocrito ni de una secreción incrementada de insulina ${ }^{(-11)}$; del mismo modo el PNA tiene concentraciones mayores de Tg y AGNE que el poblador de nivel del mar (PNM) ${ }^{(14,15)}$, relacionadas a la

${ }^{1}$ Instituto de Investigaciones Clínicas, Facultad de Medicina, Universidad Nacional Mayor de San Marcos; ${ }^{2}$ Facultad de Medicina, Universidad Nacional San Antonio Abad del Cusco, ${ }^{3}$ Academia Nacional de Medicina.

"Trabajo realizado con soporte de la Fundación Instituto Hipólito Unanue (FIHU). 
menor disponibilidad de glucosa y a una desviación preferente del metabolismo energético hacia los lípidos, a su vez vinculada a la de las hormonas hiperglicemientes como la somatotropina y glucagon $^{(16-19)}$.

\section{Material y métodos}

Se incluyó 39 obesos de altura-OA- (Cusco, Perú, 3,395 metros sobre el nivel del mar-msnm-; presión barométrica 510 y presión parcial de $\mathrm{O}^{2} 106.7 \mathrm{mmHg}$ ) y como grupo control a 35 normales de altura -NA- y a 35 obesos de nivel del marONM-(Lima, Perú, $150 \mathrm{msnm}$; presión barométrica 750 y presión parcial de $\mathrm{O}^{2} 150 \mathrm{mmHg}$ ), de ambos géneros. Los criterios de inclusión fueron 40 a 70 años de edad, las mujeres postmenopáusicas, sin antecedentes personales de hipertensión arterial ni diabetes mellitus, y como criterios de exclusión: padecer de enfermedades tiroideas, cardiovasculares, renales y hepáticas (Tabla 1). En ayunas se midió glucosa (G), triglicéridos $(\mathrm{Tg})$ colesterol total (CT) y HDL por métodos enzimáticos convencionales, insulina por RIA, ácidos grasos no esterificados (AGNE) por el método de Duncombe ${ }^{(20)}$, el

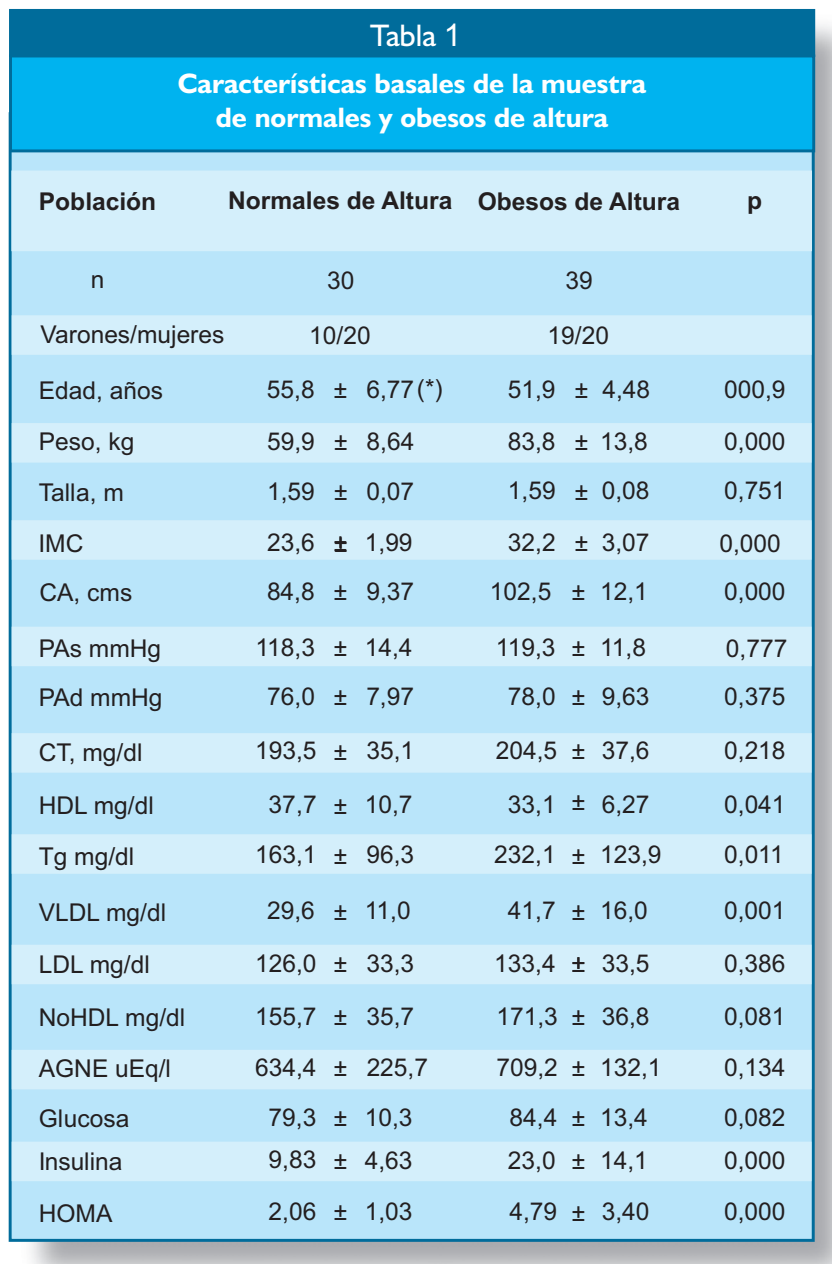

$\left({ }^{*}\right)$ Promedio \pm 1 desviación estándar de la media. colesterol VLDL, LDL y No-HDL fueron calculados. Se administró un desayuno estándar consistente en $80 \mathrm{~g}$ de pan, $30 \mathrm{~g}$ de mantequilla, 1 huevo y una infusión de manzanilla con $10 \mathrm{~g}$ de azúcar, que contiene $45 \mathrm{~g}$ de grasas $(55.4 \%$ del valor calórico total), $68 \mathrm{~g}$ de hidratos de carbono (37.2\%) y $13.4 \mathrm{~g}$ de proteínas $(7.34 \%)$ que en conjunto proporciona $730 \mathrm{Kcal}^{(21)} \mathrm{y}$ luego se midió G, I, Tg y NEFA durante 6 horas después de la ingesta alimenticia. Para los cálculos estadísticos se empleó el programa SSPS v. 23 para evaluar las diferencias de los promedios de muestras independientes ( $t$ de Student).

\section{Resultados}

En la tabla 1 se muestra las características generales de la muestra correspondiente a los normales y obesos de altura, en la no hubo diferencias en cuanto a la talla, Pas, PAd, CT, LDL, NoHDL, AGNEni G.

En la tabla 2 se muestran las características basales de los obesos de altura y de nivel del mar, en las hubo diferencias en la edad, IMC, CAy AGNE.

\begin{tabular}{lcccc|}
\multicolumn{5}{c}{ Tabla 2} \\
\multicolumn{4}{c}{$\begin{array}{c}\text { Características basales de la muestra } \\
\text { de obesos de altura y de nivel del mar }\end{array}$} \\
\hline Población & Obesos de Altura & $\begin{array}{c}\text { Obesos de } \\
\text { Nivel del Mar }\end{array}$ & p \\
\multicolumn{1}{c}{ n } & $30-39$ & 35 & \\
Varones/mujeres & $19 / 20$ & $18 / 17$ & \\
Edad, años & $51,9 \pm 4,48\left(^{*}\right)$ & $56,7 \pm 6,98$ & 000,1 \\
Peso, kg & $83,8 \pm 13,8$ & $87,2 \pm 21,9$ & 0,438 \\
Talla, m & $1,59 \pm 0,08$ & $1,57 \pm 0,08$ & 0,191 \\
IMC & $32,2 \pm 3,07$ & $35,9 \pm 6,96$ & 0,028 \\
CA, cms & $102,5 \pm 12,1$ & $110,6 \pm 11,2$ & 0,006 \\
PAs mmHg & $119,3 \pm 11,8$ & $121,3 \pm 15,4$ & 0,561 \\
PAd mmHg & $78,0 \pm 9,63$ & $74,0 \pm 10,2$ & 0,106 \\
CT, mg/dl & $204,5 \pm 37,6$ & $203,5 \pm 42,3$ & 0,915 \\
HDL mg/dl & $33,1 \pm 6,27$ & $36,0 \pm 8,60$ & 0,101 \\
Tg mg/dl & $232,1 \pm 123,9$ & $192,5 \pm 92,8$ & 0,123 \\
VLDL mg/dl & $41,7 \pm 16,0$ & $35,3 \pm 14,6$ & 0,087 \\
LDL mg/dl & $133,4 \pm 33,5$ & $131,6 \pm 39,6$ & 0,841 \\
NoHDL mg/dl & $171,3 \pm 36,8$ & $167,6 \pm 41,3$ & 0,687 \\
AGNE uEq/l & $709,2 \pm 132,1$ & $520,5 \pm 143,4$ & 0,000 \\
Glucosa & $84,4 \pm 13,4$ & $85,0 \pm 16,7$ & 0,856 \\
Insulina & $23,0 \pm 14,1$ & $22,1 \pm 17,7$ & 0,803 \\
HOMA & $4,79 \pm 3,40$ & $4,23 \pm 3,71$ & 0,504 \\
\hline
\end{tabular}

$\left({ }^{*}\right)$ Promedio \pm 1 desviación estándar de la media. 

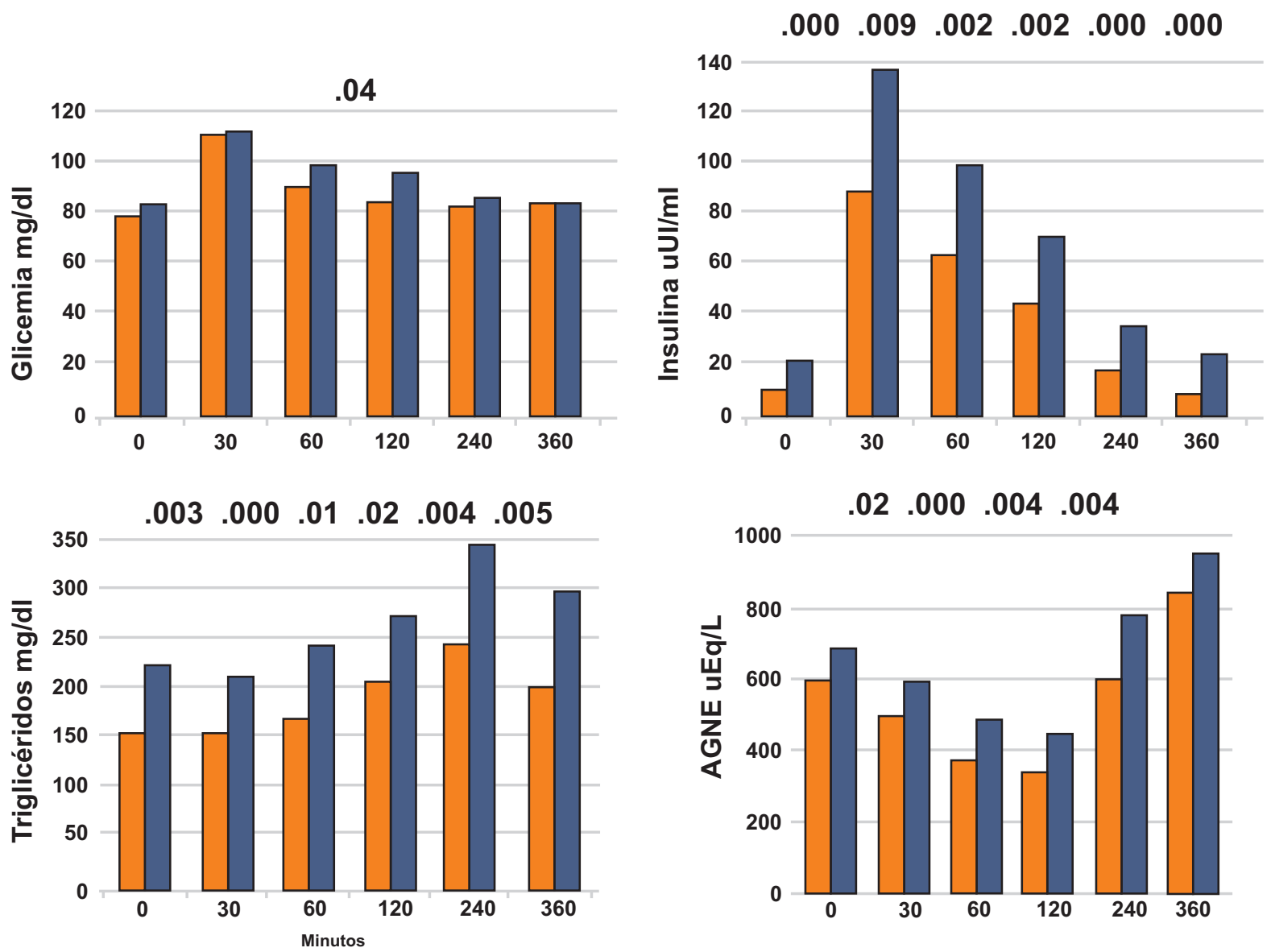

Normales

Figura 1. Comparación de los resultados entre normales y obesos de altura en el período postprandial.

En el período postprandial, los OA presentaron niveles de insulina, Tg y AGNE mucho más altos que los NA con valores de glucosa prácticamente similares que, en conjunto y los valores bajos de HDL, conforman el cuadro de resistencia a la insulina (Figura 1).

En la figura 2 se encuentran las diferencias entre obesos de nivel del mar y altura, Los OA en comparación a los ONM presentaron concentraciones más altas de Tg y AGNE, las diferencias de glicemia e insulinemia fueron menores (Figura 2).

\section{Discusión}

En el presente trabajo, se ha estudiado el metabolismo intermediario tanto en condiciones basales como en el período postprandial, este último después de la ingestión de una sobrecarga alimenticia mixta estándar, más cercana a la forma usual como se alimentan las personas que cuando se administra solo grasas ${ }^{(22)}$ o hidratos de carbono ${ }^{(23)}$. El contenido proteico, por lo tanto de aminoácidos, proporciona un estímulo adicional a la producción pancreática de insulina ${ }^{(24,25)}$ que puede significar diferencias con los resultados de otras investigaciones.
Para evaluar con mayor propiedad los resultados obtenidos en esta investigación, se debe tener presente que el sujeto normal de altura tiene cifras más bajas de glucosa y más altas de triglicéridos y AGNE que los normales de nivel del mar tanto en ayunas ${ }^{(3-15)}$ como en el período postprandial ${ }^{(1.2)}$, fenómenos que están vinculados a la hipoxia, condición que facilita el transporte y utilización de la glucosa ${ }^{(26,27)}$ y deriva el metabolismo energético a una mayor utilización de los lípidos ${ }^{(28)}$. En los obesos de altura se ha encontrado niveles más bajos de colesterol HDL y más altos de insulina, triglicéridos y AGNE que los normales de altura que, al igual de lo que sucede en obesos de nivel del mar ${ }^{(29)}$, constituyen rasgos bioquímicos inequívocos del síndrome resistencia a la insulina, esta vez, en condiciones de hipoxia.

Los obesos de altura en comparación a los obesos de nivel del mar presentaron cifras más altas de triglicéridos y AGNE, que están relacionadas a la concurrencia de 2 fenómenos; por un lado, como se ha mencionado, la obesidad per se incrementa las concentraciones triglicéridos y AGNE (29,30-32) y, por otro, la hipoxia de la exposición a la altura facilita el transporte y utilización de la glucosa ${ }^{(26,27)}$ y deriva el metabolismo energético a una mayor utilización de los lípidos ${ }^{(28)}$. 

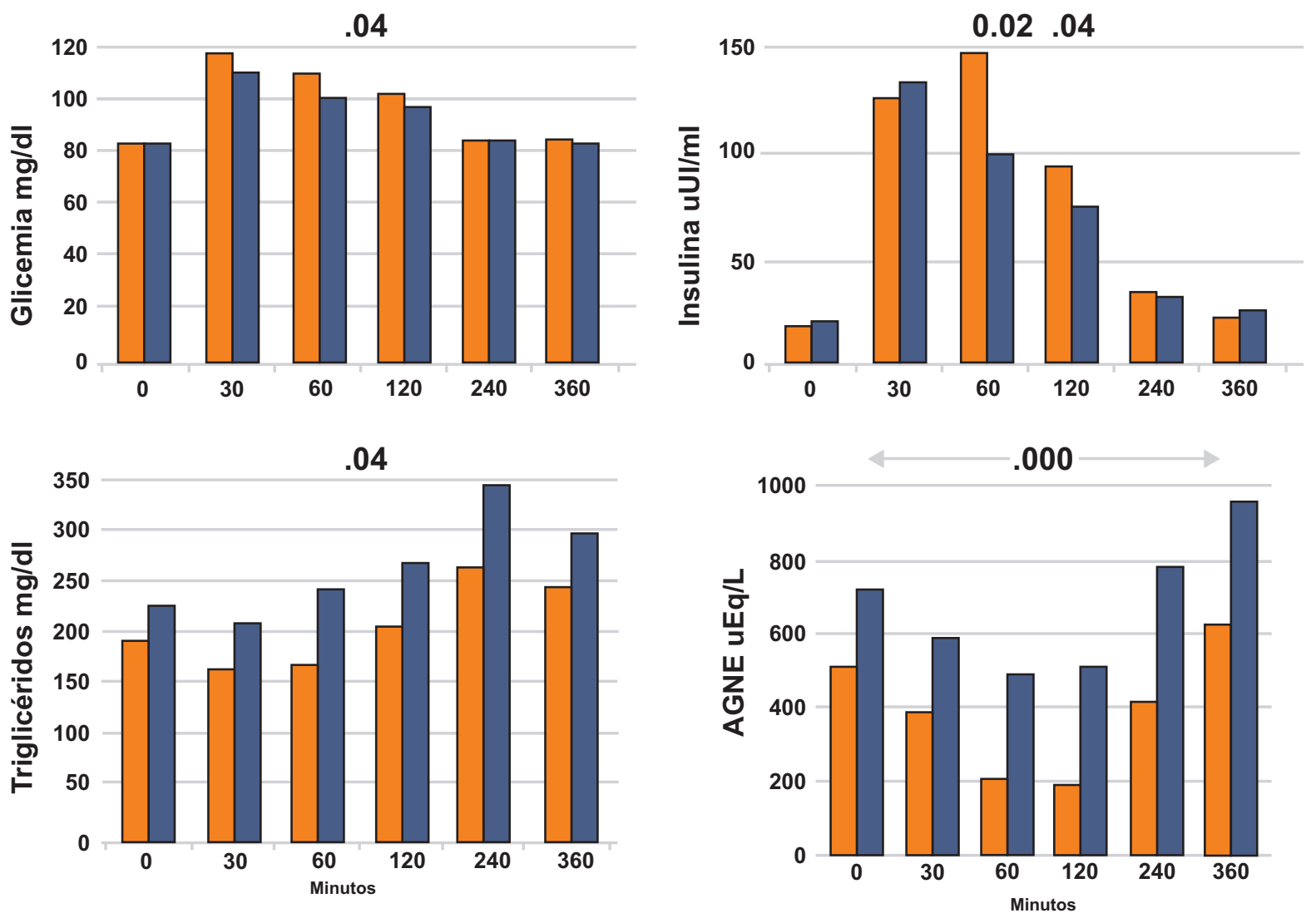

Nivel del mar

Altura

Figura 2. Comparación de los promedios de los marcadores bioquímicos basales y en el período postprandial entre obesos de nivel del mar y de altura.

Estos resultados demuestran que la obesidad en los pobladores de altura también constituye una condición patológica que incrementa el riesgo a desarrollar alteraciones cardiovasculares y todas las comorbilidades inherentes a esta patología.
Referencias bibliográficas

1. Garmendia F, Pando R, Mendoza Y, Torres W, Urdanivia E. Basal and postprandial intermediary metabolism in normal male high altitude dwellers. MOJ Biology and Medicine 2018; 3(1):4-6.

2. Garmendia F, Pando R, Mendoza Y, Torres W. Metabolismo intermediario basal y postprandial en mujeres normales de altura. En prensa.

3. Forbes WH. Blood sugar and glucose tolerance at high altitude, Am J Physiol 1936;116:309-16.

4. San Martín M. Distribución de la glucosa sanguínea y su variación en el cambio de altitud, An Fac Med Lima 1940;23: 312.

5. Monge CC. Glucosa, acido láctico y acido pirúvico a nivel del mar y altura. An Fac Med,Lima 1949;32:1.

6.Picón-Reátegui E. Studies on the metabolism of carbohydrates at sea level and at high altitude. Metabolism 1962;11:1148-54.
7. Picón-Reátegui E. Intravenous glucose tolerance test at sea level and at high altitude. J Clin Endocrinol Metab 1963;23: 1256-61.

8. Calderon R, Llerena L. Carbohydrate metabolism in people living in chronic hypoxia. Diabetes 1965;14:100-5.

9. Garmendia F, Arroyo J, Muro M. Glicemia del nativo normal de altura. Arch Inst Biol Andina 1970;3:209-16.

10. Garmendia F, Torres J, Tamayo R, Urdanivia E. Aportes al conocimiento de la glicemia de altura. Arch Inst Biol Andina 1972;5(1):51-6.

11. Garmendia F, Urdanivia E, Torres J, Tamayo R. Carbohydrate metabolism at high altitude. VIII Congress of the International Diabetes Federation, Abstr. N ${ }^{\circ} 262$, Brussels, Belgium, 1973.

12. Sutton J, Garmendia F. Variaciones hormonales durante el esfuerzo físico en la altura. Arch Biol Andina, 1977;7:83-93

13. Castillo O, Woolcott $\mathbf{O}$, Gonzales E, Tello V, Tello L, 
Villarreal C y col. Monitoreo continuo de la glicemia en el poblador de los Andes. Diagnóstico 2006;45(1):39-43.

14. Llerena LA, Muñoz JM, Muñoz T. Ácidos grasos no esterificados (AGNE) en suero de gestantes, recién nacidos y hombres normales de altura. Ginec Obst 1971;17

15. Garmendia F, Jo N, Damas L, Fajardo W. Incremento de la utilización de la glucosa y trigliceridemia más alta en el adulto mayor de altura.. XIV Congreso Panamericano de Endocrinología, Cancún, México, 2 a 7 de noviembre de 1997.

16. Sutton J, Young JD. Lazarus L, Hickie JB, Garmendia F, Velásquez T. The Hormonal response to altitude. Lancet 1970; 2:1194.

17. Garmendia F, Arévalo C. Concentración normal y patológica de hormona de crecimiento en sangre. Acta Med Peruana 1975;4:8-16.

18. Gonzales GF, Coyotupa J, Guerra,García R. Elevated levels of growth hormone in natives from high altitude. Interrelationship with glucose levels. Acta Andina 1992;1:858.

19. Sutton JR, Garmendia. Hormonal responses to exercise at altitude in sea level and mountain man. En High Altitude Physiology and Medicine. Brendel W, Zink RA, Ed. Springer Verlag, New York, Heidelberg, Berlin, 1982:165-171.

20. Duncombe WG. The colorimetric micro determination of nonesterified fatty acids in plasma. Clin Chim Acta 1964;9: 122125.

21. Garmendia F, Pando R, Torres W, Valqui W, Jamieson C, Blufstein N. Metabolismo postprandial en adultos mayores normales de nivel del mar. An Fac Medicina Lima 2003;64 (2):107-111

22. Dubois C, Beaumier G, Juhel C, Armand M, Portugal H et al. Effect of graded amounts $(0-50 \mathrm{~g})$ of dietary fatt on postprandial lipemia and lipoproteins in normolipemic adults. Am J Clin Nutr 1998;67:31-8.
23. Bantle JP, Laine DC, Castle GW, Thomas JW et al. Postprandial glucose and insulin responses containing different carbohydrates in normal and diabetic subjects. N Engl J Med 1983;309:7-12.

24. Floyd JC, Fajans SS, Conn JW, Knopf RF et al. Insulin secretion in response to protein ingestion. J Clin Invest 1966; 45(9):1479-86.

25. Floyd JC, Fajans SS, Conn JW, Knopf RF, et al. Stimulation of Insulin secretion by aminoacids. J Clin Invest 1966;45(9): $1487-1502$

26. Loike JD, Cao L, Brett J, Ogawa S, et al. Hypoxia induces glucose transporter expression in endothelial cells. Am J Physiol Cell Physiol 1992;263(2):C326-C333.

27. Basham N, Burdett E, Hundal HS, Klip A. Regulation of glucose transport and GLUT1 glucose transporter expression by O2 in muscle cells in culture. Am J Physiol 1992;262(3): C682-C690.

28. Yin J, Gao Z, He Q, Zhou D, Guo ZK, Ye J. Role of hypoxia in obesity-induced disorders of glucose and lipid metabolism in adipose tissue. Am J Physiol Endocrinol Metab 2008;296: E333-E342.

29. Garmendia F, Crispín S, Flores R, y col. Estudio del metabolismo intermediario basal y postprandial en obesos. Diagnóstico 2012;51(1):7-11.

30. Reaven GM. Banting Lecture 1988. Role of insulin resistance in human disease. Diabetes 1988;37:1595-1607.

31. Carey DG, Jenkins AB, Campbell LD, et al. Abdominal fat and insulin resistance in normal and overweight women. Direct measurement reveal a strong relationship in subjects at both low and high risk of NIDDM. Diabetes 1996;45:633-38.

32. Steinberg HO, Dumbiner B: Pathophysiology of obesity and metabolic response to weight loss. In Obesity Barry Gumbiner editor, American College of Physicians, Philadelphia 2001: 5066.

Citar como: Garmendia-Lorena F. Pando-Alvarez R. Mendoza-Muñoz Y. Torres-Damas W. Metabolismo intermediario basal y postprandial en obesos de altura y nivel del mar. Diagnóstico(Lima).2019;58(2):67-71. DOI: https://doi.org/10.33734/diagnostico.v58i2.205

Correspondencia: Fausto Garmendia Lorena Dirección: Calle Elías Aguirre N 141-409, Miraflores, Lima 18 Teléfono: 981903903 Correo electrónico: garmendiafausto@gmail.com

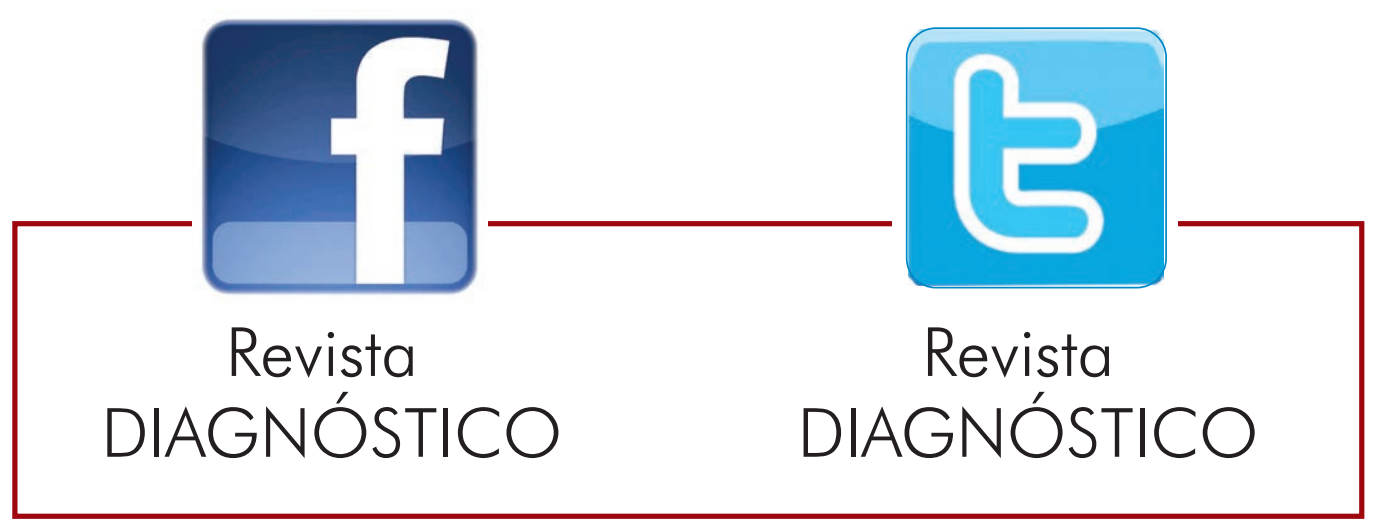

